\title{
Relação entre o nível de atividade física e atenção de alunos do ensino médio em uma escola pública do interior de Minas Gerais
}

\author{
Relationship between the level of physical activity and attention of high school students in a public \\ school in the interior of Minas Gerais \\ Relación entre el nivel de actividad física y la atención de los estudiantes de secundaria en una \\ escuela pública del interior de Minas Gerais
}

\author{
Lucas Miranda da Silva Souza \\ ORCID: https://orcid.org/0000-0001-9333-5384 \\ Centro Universitário Governador Ozanam Coelho, Brasil \\ E-mail: bernardes1322@gmail.com \\ Luciano Bernardes Leite \\ ORCID: https://orcid.org/0000-0002-0885-5834 \\ Universidade Federal de Viçosa, Brasil \\ E-mail: luciano.leite@ufv.br \\ Ana Amélia de Souza Pereira \\ ORCID: https://orcid.org/0000-0002-5434-7451 \\ Centro Universitário Governador Ozanam Coelho, Brasil \\ E-mail: ana.amelia@unifagoc.edu.br \\ Anselmo Gomes de Moura \\ ORCID https://orcid.org/0000-0002-1505-5595 \\ Centro Universitário Governador Ozanam Coelho, Brasil \\ E-mail: anselmo.moura@unifagoc.edu.br \\ Renata Aparecida Rodrigues de Oliveira \\ ORCID: https://orcid.org/0000-0002-5004-5253 \\ Centro Universitário Governador Ozanam Coelho, Brasil \\ E-mail: renata.oliveira@unifagoc.edu.br \\ Denise Coutinho de Miranda \\ ORCID: https://orcid.org/0000-0001-8714-6309 \\ Centro Universitário Governador Ozanam Coelho, Brasil \\ E-mail: denise.miranda@gmail.com \\ Victor Neiva Lavorato \\ ORCID: https://orcid.org/0000-0001-9914-4722 \\ Centro Universitário Governador Ozanam Coelho, Brasil \\ E-mail: victor.lavorato@unifagoc.edu.br
}

\begin{abstract}
Resumo
Este estudo teve como objetivo verificar e comparar o nível de atenção e o nível de atividade física em alunos do ensino médio de uma escola em Piraúba-MG. Para isso, foram avaliados adolescentes de ambos os sexos com faixa etária de 15 a 17 anos. Como instrumentos de avaliação, foram utilizados dois testes. O primeiro teste aplicado foi o Questionário Internacional de Atividade Física (IPAQ- versão curta), que busca estimar o nível de atividade física dos avaliados. Em seguida, foi utilizado o Teste de Atenção por Cancelamento (TAC), composto por atividades de cancelamento de figuras em três etapas e níveis diferentes (TAC1, TAC2, TAC3), sendo utilizado para estimar o nível de atenção, ambos testes foram aplicados de forma presencial. Os resultados indicaram um melhor desempenho no teste de atenção dos alunos que foram classificados como Ativos, principalmente na primeira parte do teste (TAC1), com um melhor tempo e um menor percentual de erros. Assim, conclui-se que adolescentes ativos obtiveram um melhor resultado em uma parte específica do teste (TAC1), no que diz respeito ao nível de atenção seletiva.
\end{abstract}

Palavras-chave: Atividade física; Atenção; Adolescentes; Ensino.

\begin{abstract}
This study aimed to verify and compare the level of attention and the level of physical activity in high school students from a school in Piraúba-MG. For this, adolescents of both sexes aged 15 to 17 years were evaluated. As assessment instruments, two tests were used. The first test applied was the International Physical Activity Questionnaire (IPAQshort version), which seeks to estimate the level of physical activity of the individuals evaluated. Then, the Attention Cancellation Test (CAT) was used, consisting of activities to cancel pictures in three stages and different levels
\end{abstract}


(TAC1, TAC2, TAC3), being used to estimate the level of attention, both tests were applied from in person. The results indicated a better performance in the attention test of students who were classified as Active, mainly in the first part of the test (TAC1), with a better time and a lower percentage of errors. Thus, it is concluded that active adolescents obtained a better result in a specific part of the test (TAC1), with regard to the level of selective attention. Keywords: Physical activity; Heads up; Teenagers; Teaching.

\section{Resumen}

Este estudio tuvo como objetivo verificar y comparar el nivel de atención y el nivel de actividad física en estudiantes de secundaria de una escuela de Piraúba-MG. Para ello, se evaluaron adolescentes de ambos sexos de 15 a 17 años. Como instrumentos de evaluación se utilizaron dos pruebas. La primera prueba aplicada fue el Cuestionario Internacional de Actividad Física (IPAQ- versión corta), que busca estimar el nivel de actividad física de los individuos evaluados. Luego, se utilizó la Prueba de Cancelación de Atención (CAT), que consiste en actividades para cancelar fotografías en tres etapas y diferentes niveles (TAC1, TAC2, TAC3), siendo utilizado para estimar el nivel de atención, ambas pruebas se aplicaron de manera presencial. Los resultados indicaron un mejor desempeño en la prueba de atención de los estudiantes que fueron clasificados como Activos, principalmente en la primera parte de la prueba (TAC1), con un mejor tiempo y un menor porcentaje de errores. Así, se concluye que los adolescentes activos obtuvieron un mejor resultado en una parte específica de la prueba (TAC1), en cuanto al nivel de atención selectiva.

Palabras clave: Actividad física; Aviso; Adolescentes; Enseñanza.

\section{Introdução}

Sabe-se que a prática regular de atividade física está diretamente relacionada a prevenção de doenças não transmissíveis e pode promover diversos benefícios físicos ou psicológicos em indivíduos de diversas faixas etárias (Cassidy et al., 2004; Mummery et al., 2004; Olson et al., 2005; Chyun et al., 2006). Dessa forma, as escolas são locais ideais para gerar um aumento na prática de atividade física, com diversas oportunidades para que os jovens se tornem fisicamente ativos durante a semana, como intervalos, esportes, aulas e viagens ativas, ou seja, intervenções direcionadas a esses períodos podem levar a uma contribuição de 50\% para atender as diretrizes de atividade física (Watson et al., 2017).

Para que se torne possível alcançar a meta de desenvolvimento, é essencial que os níveis de atividade física aumentem em todas as faixas etárias, mas principalmente entre crianças e adolescentes. Porém, o número de jovens que praticam atividade física vem caindo devido ao aumento dos comportamentos sedentários (Barbosa et al., 2020).

As recomendações de atividade física citadas por Barbosa et al. (2020), de acordo com a Organização Mundial da Saúde (OMS) é que adolescentes e crianças de 5 a 17 anos atinjam no mínimo 60 minutos de atividade física por dia. Tendo em vista que o cumprimento dos níveis de atividades físicas diárias é fundamental para o desenvolvimento de habilidades cognitivas, motoras, sociais, e para uma boa saúde musculoesquelética. Segundo a Organização Mundial da Saúde (OMS) citado por Hall et al. (2021), com a chegada da pandemia do COVID-19, houve uma maior dificuldade de acesso a atividade física, com isso, cerca de $31 \%$ das pessoas com idade entre 15 anos ou mais, são fisicamente inativas e aproximadamente 3,2 milhões de mortes por ano são atribuídas a esse comportamento pouco saudável.

Quando se fala da prática de atividade física no Brasil, a porcentagem de adolescentes praticantes de atividade física regular antes da pandemia era de 28,7\% e durante a pandemia do COVID-19 esse número diminuiu para 15,74\%. Essa diminuição ocorreu em ambos os sexos e faixas etárias, levando também ao aumento no sedentarismo de 44,57\% para 70,15\% em relação aos adolescentes (Malta et al., 2020).

No entanto, com a pandemia do COVID-19, o processo para que se tenha um aumento na porcentagem de pessoas praticantes de atividade física tem sido lento e de forma gradativa. Em todo mundo, o número de pessoas que seguem as recomendações globais de atividade física está baixo, 1 em cada 4 adultos e 3 em cada 4 adolescentes (com idades entre 11-17 anos) não seguem as recomendações. Com isso o nível de inatividade física alcançou cerca de $70 \%$ em alguns países, devido ao excesso da tecnologia e mudanças nos padrões de transporte e urbanização, levando o aumento de diversas condições adversas de saúde, como a morbidade, mortalidade, inatividade física e comportamento sedentário, representando um fardo 
econômico em todo mundo (Amini et al., 2021).

Parte do efeito da atividade física no desempenho acadêmico é mediado pelas funções executivas do cérebro, com indução do crescimento neural e modificação na transmissão sináptica, resultando em mudanças no pensamento, tomada de decisão e níveis de atenção. A atividade física aguda aumenta a excitação fisiológica, levando também à elevação da atenção, disparando a liberação de neurotransmissores que acarretam melhoria nos processos cognitivos. Já a atividade aeróbica, aumenta a aptidão cardiovascular, gera melhora na função cerebral, em áreas específicas pela aprendizagem e memória. Além disso, a atividade física regular é responsável por contribuir no desempenho acadêmico por meio da autopercepção positiva, regulação emocional e funcionamento cognitivo (Barbosa et al., 2020).

Dentre as fases importantes da vida com relação a atividade física, a adolescência é um período conhecido por uma fase que caracteriza diversos tipos de desenvolvimentos, como o crescimento físico e maturação cerebral, mas também é conhecido por uma etapa onde existe a predominância de comportamentos não saudáveis, como a inatividade física por exemplo. Consequentemente, a inatividade física gera fatores negativos nos adolescentes, por isso, qualquer vantagem que possa ajudar na saúde e melhoria no desempenho acadêmico de um aluno do ensino médio pode ser útil, sendo então, importante verificar como a redução do nível de atividade física durante a pandemia pode afetar no nível de atenção dos alunos do Ensino Médio. Levando em consideração a importância e certeza de que o controle cognitivo foi estabelecido como um fator determinante do desempenho acadêmico (Dubuc et al., 2019). Neste contexto, o objetivo do estudo foi verificar e comparar estudantes com diferentes níveis de atividade física e o nível de atenção dos mesmos em uma escola em PiraúbaMG.

\section{Metodologia}

\section{Amostra}

A amostra foi composta por 21 adolescentes, sendo 15 homens e 6 mulheres, com idade de 15 a 17 anos, de uma escola pública em Piraúba, Minas Gerais. Foram excluídos do estudo os participantes que não apresentaram o Termo de Assentimento ou não responderam a todas as perguntas dos questionários. Para participar do estudo, os estudantes deveriam estar regularmente matriculados na escola a pelo menos 1 ano.

\section{Instrumentos}

\section{Nivel de Atividade Física}

Para avaliar o nível de atividade físico dos adolescentes foi utilizado o Questionário Internacional de Atividade Física (IPAQ- versão curta, que busca estimar o nível de atividade física dos avaliados, classificando-os em "Ativos" (ativos e muito ativos) e "Não ativos" (sedentários e insuficientemente ativos A e B). Este questionário é composto por 4 perguntas, contendo alternativas A e B, que estão relacionadas ao tempo que a pessoa gastou realizando atividade física na semana (Matsudo et al., 2001). A classificação dos dados obtidos separa e conceitua as categorias em: Sedentário (indivíduo que não realiza nenhuma atividade física por pelo menos 10 minutos contínuos durante a semana); Irregularmente Ativo (indivíduo que realiza atividade física, porém, de maneira insuficiente para ser classificado como ativo. Para realizar essa classificação soma-se a frequência e a duração das diferentes atividades (caminhada + moderada + vigorosa), esta categoria divide-se em dois grupos): Irregularmente Ativo A (indivíduo que atinge pelo menos um dos critérios de recomendação quanto à frequência (5 dias/semana) ou duração (150 minutos/semana) da atividade) e Irregularmente Ativo B (indivíduo que não alcançou nenhum dos critérios da recomendação quanto à duração ou frequência de atividade); Ativo (indivíduo que atingiu as recomendações de atividade vigorosa ( 3 dias ou mais/semana e 20 minutos ou mais/sessão) ou atividade moderada ou caminhada ( 5 dias ou 
mais/semana e 30 minutos ou mais/sessão) ou qualquer atividade somada (caminhada + moderada + vigorosa) em 5 dias ou mais/semana e 150 minutos ou mais/semana); Muito Ativo (indivíduo que cumpriu as recomendações de atividade vigorosa (5 dias ou mais/semana e 30 minutos ou mais/sessão), atividade vigorosa ( 3 dias ou mais/semana e 20 minutos ou mais/sessão + moderada e/ou caminhada em 5 dias ou mais/semana e 30 minutos ou mais/sessão).

\section{Nivel de atenção}

Para avaliar o nível de atenção dos adolescentes foi utilizado o Teste de Atenção por cancelamento (TAC) que consiste em três matrizes impressas com diferentes estímulos, sendo a tarefa do avaliado assinalar os estímulos iguais ao estímulo alvo que foram apresentados previamente, em um tempo de 1 minuto para cada etapa (Tortella, 2008). Na primeira parte do teste foi avaliada a atenção seletiva, ou seja, a capacidade do avaliado de selecionar estímulos-alvo entre outros estímulos distratores (TAC1). Para isso, é utilizada a prova de cancelamento de figuras, tendo uma matriz contendo impressos seis estímulos diferentes (círculo, quadrado, triângulo, cruz, estrela e traço). As figuras são de cor preta em fundo branco, distribuídos em 18 linhas, sendo que cada linha é composta por 20 figuras resultando em um total de 360 estímulos. Os avaliados terão de marcar os estímulos fornecidos igualmente ao estímulo-alvo que estará na parte superior da matriz (Figura $1)$.

Figura 1. Ilustração da primeira parte do Teste de Atenção por Cancelamento cujo objetivo é avaliar a atenção seletiva.

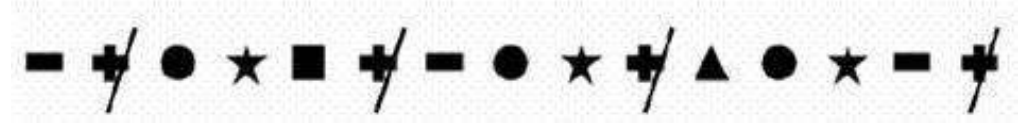

Fonte: Tordella (2018).

Na segunda parte ainda foi avaliada a atenção seletiva, porém a prova irá conter um grau de dificuldade maior (TAC2). O estímulo-alvo nesta etapa será composto por duas figuras impressas na parte superior da folha e 18 linhas contendo 20 figuras cada para a marcação, com 360 no total, assim como na primeira parte (Figura 2).

Figura 2. Ilustração da segunda parte do Teste de Atenção por Cancelamento cujo objetivo é avaliar a atenção seletiva.

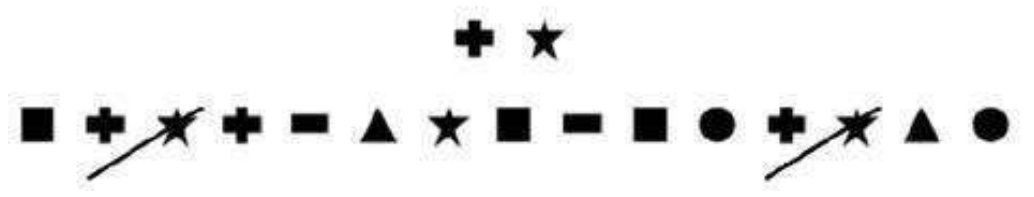

Fonte: Tordella (2018).

Por fim, na terceira parte do teste o objetivo foi avaliar a atenção alternada, ou seja, a capacidade do indivíduo de mudar o foco de atenção de tempos em tempos, sendo utilizado também o teste de cancelamento de figuras (TAC3). O teste terá o estímulo-alvo alternado de linha em linha, sendo a primeira figura de cada linha o estímulo-alvo. Nesta parte como as demais os indivíduos terão 18 linhas com 20 figuras cada, totalizando também 360 figuras, porém as ordens serão diferentes (Figura 3). 
Figura 3. Exemplo da terceira parte do Teste de Atenção por Cancelamento, cujo objetivo é avaliar a atenção seletiva.

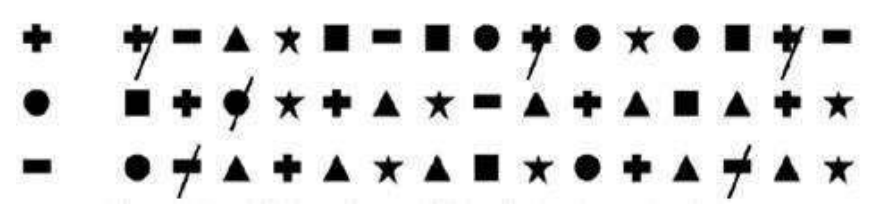

Fonte: Tordella (2018).

\section{Análise estatística}

Os dados foram apresentados como média e desvio padrão da média ou porcentagem simples. Os grupos de estudantes ficaram divididos em "Ativos" (ativos + muito ativos) e "Não ativos" (sedentários + insuficientemente ativos A e B). Inicialmente foi realizado um teste para verificação da normalidade dos dados. Posteriormente, foi utilizado um teste t de Student para comparação dos dados de atenção. O nível de significância adotado teve a porcentagem de 5\%. Os dados foram analisados através do programa estatístico Graph Pad Prism 8.0®.

\section{Resultados}

A Tabela 1 apresenta os resultados referentes ao nível de atividade física dos avaliados. Observa-se que 38,09\% da amostra foi classificada como "Muito Ativo", seguido de "Irregularmente Ativo A (33,33\%)", "B (14,28)" e "Ativo (14,28\%)".

Tabela 1 - Nível de atividade física dos participantes.

\begin{tabular}{lc}
\hline \multicolumn{1}{c}{ Nível de Atividade Física } & \% \\
\hline Muito Ativo & 38,09 \\
Ativo & 14,28 \\
Irregularmente Ativo A & 33,33 \\
Irregularmente Ativo B & 14,28 \\
Sedentário & 0 \\
\hline
\end{tabular}

Fonte: Dados da pesquisa (2021).

A Tabela 2 apresenta os resultados relativos ao (TAC), tanto em relação a cada fase do teste, divididas em TAC 1, TAC 2 e TAC 3, bem como em relação ao total. Observa-se que no TAC 1 a média referente ao teste foi de 41,86, seguindo de 3,79 no TAC 2 e 61,05 no TAC 3.

Tabela 2 - Teste de atenção por cancelamento.

\begin{tabular}{lcc}
\hline & Teste de Atenção & $\begin{array}{c}\text { Média e Desvio } \\
\text { Padrão }\end{array}$ \\
\hline TAC 1 & $41,81 \pm 5,76$ \\
TAC 2 & $3,79 \pm 1,17$ \\
TAC 3 & $61,05 \pm 3,47$ \\
TOTAL & $108,7 \pm 5,78$ \\
\hline
\end{tabular}

Fonte: Dados da pesquisa (2021)

A Figura 4 apresenta o desempenho dos avaliados classificados como ativos (ativos + muito ativos) e inativos (irregularmente ativo A e B + sedentários) para cada fase do TAC. Foi possível observar que no TAC 1 o desempenho dos ativos foi maior em relação aos inativos $(\mathrm{p}=0$,0455) (Figura 4A). Não houve diferença para TAC 2 e TAC 3 (p > 0,05) (Figura 4B e 4C) entre os grupos. 
Figura 4. Número de acertos no TAC 1 (A), TAC 2 (B) e TAC 3 (C) para os avaliados ativos e inativos.

A

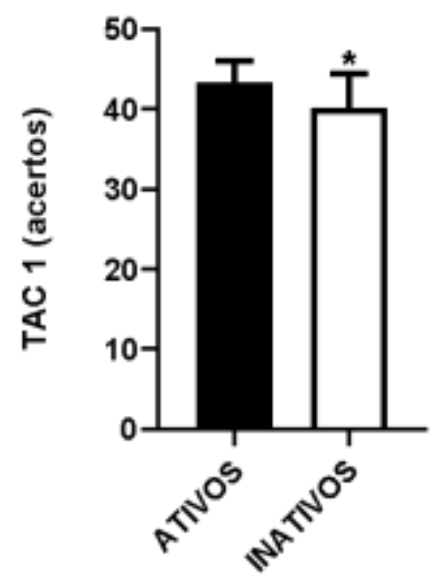

B

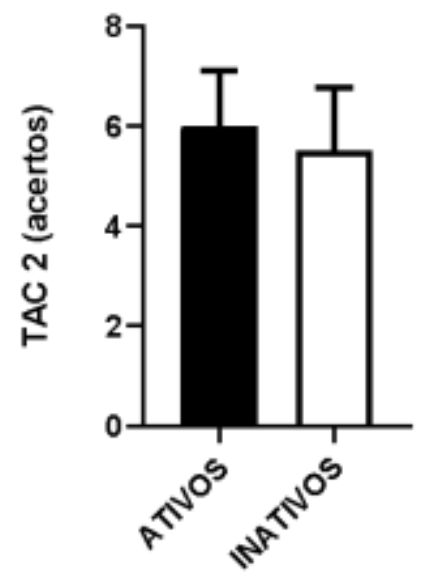

C

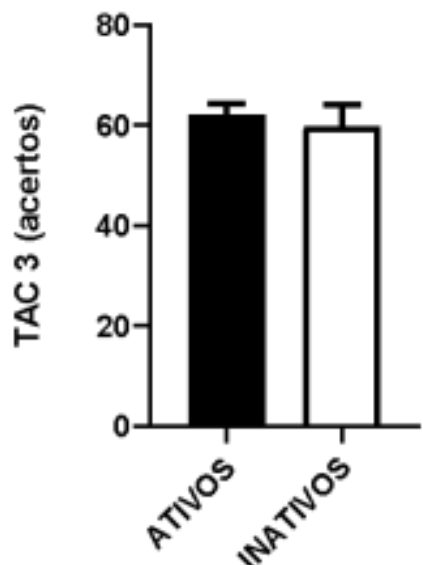

Fonte: Dados da pesquisa (2021)

A Figura 5 apresenta o desempenho total dos avaliados ativos e inativos no TAC. Foi observado que o tempo de resposta para os indivíduos inativos foi maior $(\mathrm{p}=0,0075)$ (Figura 5A) e o número de acertos foi menor $(\mathrm{p}=0,0095)($ Figura 5B) que os indivíduos ativos.

Figura 5. Tempo para a realização do TAC (A) e número de acertos no TAC (B) para os avaliados ativos e inativos.

A

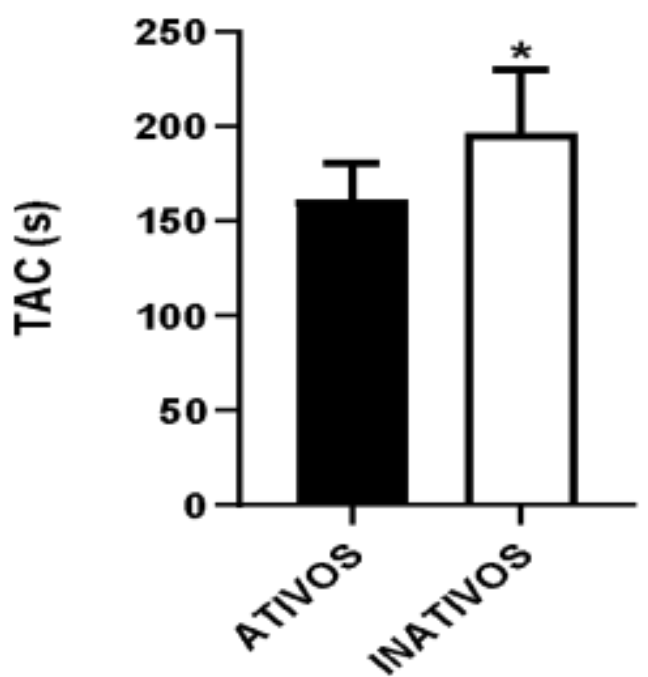

B

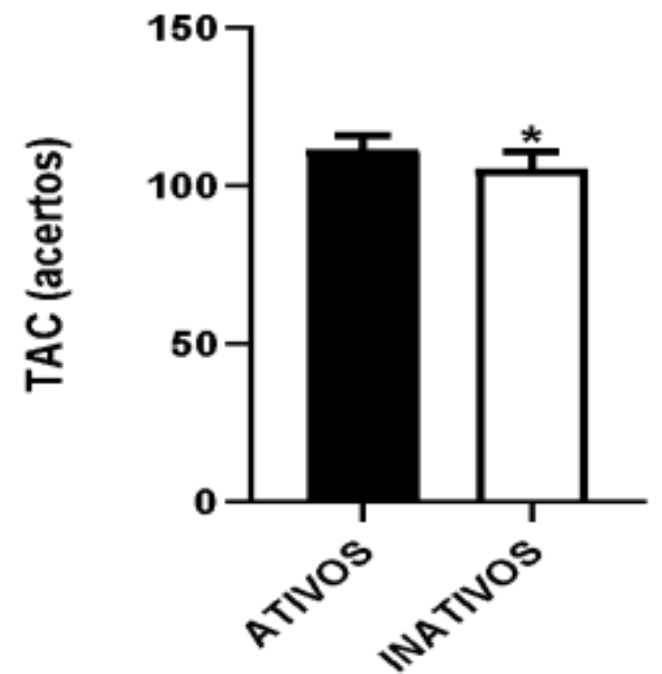

Fonte: Dados da pesquisa (2021)

\section{Discussão}

O presente estudo teve como objetivo investigar e comparar os diferentes níveis de atividade física com os níveis atenção em adolescentes em uma escola pública de Piraúba-MG. O principal achado deste estudo mostra que os avaliados que foram classificados como ativos e muito ativos tiveram um melhor desempenho no teste de atenção por cancelamento, principalmente na primeira parte do teste (TAC1), o qual avalia a atenção seletiva do avaliado.

A diferença encontrar no TAC1 pode estar associada ao tipo de atenção cobrada nessa parte do teste, exigindo mais atenção do avaliado, já que em meio aos estímulos alvos, havia estímulos de distração. Mostrando assim, que a prática de 
atividade física regular pode estar interferindo de maneira positiva no melhor desempenho dessa parte do teste.

Já o desempenho dos dois grupos em relação à média de acertos no teste de atenção por cancelamento (TAC), foi obtido uma média de acertos de 41,81 em 49 figuras na primeira parte do teste (TAC 1), 3,79 em 7 figuras na segunda parte do teste (TAC 2), 61,05 em 67 figuras na terceira parte do teste (TAC 3), e por fim, uma média de 108,7 em 123 figuras no total das três etapas. De acordo com Freire et al. (2014), a prática regular de atividade física é fundamental em qualquer idade e tem sido considerada um meio de preservar e melhorar a saúde geral do indivíduo, incluindo processos cognitivos. Observando que esses processos podem interferir de forma direta no TAC, pode-se considerar sua relação com os níveis de atenção e, consequentemente, com os resultados obtidos.

Estudos envolvendo atividade física e saúde em adolescentes mostraram os inúmeros benefícios da prática regular nessa faixa etária, dentre eles destacam-se: melhora na composição óssea, no perfil lipídico, no perfil metabólico, redução do percentual de gordura corporal, aumento do desempenho escolar, melhora significativa na saúde física, mental, bem-estar psicossocial (Hallal et al., 2005; Pucci et al., 2012; Li et al., 2017).

De acordo com Gomez-Pinnila e Hillman (2013), a atividade física se faz eficaz na melhoria da saúde cognitiva ao longo de toda vida humana, tendo a aptidão aeróbica como forma de evitar a perda de tecido cerebral e de aspectos funcionais das regiões de ordem superior, envolvidas no controle da cognição, fazendo com que indivíduos mais ativos sejam capazes de alocar maiores recursos de níveis de atenção para o ambiente e de processar informações mais rapidamente. Essas informações tornam-se coerentes e podem explicar o motivo de um melhor resultado dos avaliados ativos em relação ao tempo de execução do teste e um melhor aproveitamento em relação aos acertos.

Além desses benefícios gerados pela atividade aeróbia, o exercício físico agudo, segundo Barbosa et al. (2020), aumenta a excitação fisiológica, levando também à elevação da atenção, disparando a liberação de neurotransmissores que acarretam melhoria nos processos cognitivos.

Por outro lado, comportamentos baseados em amplo tempo de tela, são atividades de lazer muito utilizadas pelos jovens, principalmente com a chegada da pandemia da Covid-19, como por exemplo, assistir televisão e usar sites de rede social. Esse tempo cumulativo empregado nesses comportamentos é frequentemente considerado um risco para essa faixa etária, estimando que entre $40 \%$ a $80 \%$ dos jovens excedem às recomendações de tempo de tela, podendo se tornar jovens insuficientemente ativos, gerando um declínio na saúde cognitiva (Yan et al., 2017).

De acordo com Erickson et al. (2019), a prática regular de atividade física auxilia também no desempenho acadêmico e testes neuropsicológicos, como os que medem a velocidade de processamento, memória e função executivas. Situações de atividade física moderada a vigorosa trazem benefícios transitórios para a cognição durante o período pós-recuperação, tendo assim, fortes evidências que maiores níveis de atividade física estão associados a um risco reduzido de desenvolver comprometimento cognitivo, sendo essa relação baseada em princípios neurobiológicos.

Um estudo realizado por Sibley e Etnier (2013), obteve uma relação positiva entre função cognitiva e atividade física em crianças e adolescentes com idades entre 4 a 18 anos, seguindo o conceito de que a atividade física pode estar relacionada ao desenvolvimento da cognição. Os resultados revelaram que a participação em atividades físicas estava relacionada ao desempenho cognitivo ao longo de oito categorias de medição, sendo citadas habilidades perceptivas, quociente inteligente, desempenho, testes verbais, testes de matemática, memória, nível de desenvolvimento, prontidão acadêmica, entre outros. Obtendo resultados que indicaram uma relação benéfica da atividade física em todas as categorias cognitivas. $\mathrm{O}$ resultado do estudo acima mostra diversas habilidades perceptivas que obtiveram melhorias, este fato pode mostrar também um melhor resultado dos alunos ativos, tendo em vista que grande parte dessas habilidades estavam no TAC.

Diante dos resultados do presente estudo, e de outros associados à interferência da atividade física nos níveis de atenção, a hipótese deste estudo foi parcialmente atingida, visto que, embora a TAC 2 e TAC 3 não apresentaram diferença 
significativa, a TAC 1 mostrou uma diferença significativa entre os dois grupos, com um melhor desempenho do grupo de avaliados ativos.

Ressalta-se que esta pesquisa apresentou fatores limitantes, como o número de avaliados, devido ao fechamento da escola pela pandemia da Covid-19, sendo assim, a coleta feita nas casas e serviços dos avaliados, o que dificultou para um maior número de dados.

\section{Considerações Finais}

O estudo mostra que adolescentes classificados como ativos obtiveram melhores resultados em comparação aos demais alunos classificados como inativos, no que diz respeito ao nível de atenção, especificamente no (TAC1), sendo essa parte, responsável por avaliar a atenção seletiva dos avaliados.

A partir desses dados, faz-se necessário reforçar o estímulo à prática de atividades físicas, a fim de proporcionar benefícios fisiológicos em vários âmbitos, como, por exemplo, nos processos cognitivos.

Devido ao baixo número de avaliados, sugere-se que novos estudos comparando os diferentes níveis de atividade física dos jovens com o nível de atenção seja feito com um maior número de dados, o que poderia implicar em um resultado mais abrangente e fidedigno.

\section{Referências}

Amini, H., Habibi, S., Islamoglu, A. H., Isanejad, E., Uz, C., \& Daniyari, H. (2021). COVID-19 pandemic-induced physical inactivity: the necessity of updating the Global Action Plan on Physical Activity 2018-2030. Environmental Health and Preventive Medicine, 26(1), 1-3.

Barbosa, A., Whiting, S., Simmonds, P., Scotini Moreno, R., Mendes, R., \& Breda, J. (2020). Physical activity and academic achievement: An umbrella review. International Journal of Environmental Research and Public Health, 17(16), 5972.

Cassidy, K., Kotynia-English, R., Acres, J., Flicker, L., Lautenschlager, N. T., Almeida, O. P., \& Almeida, O. P. (2004). Association between lifestyle factors and mental health measures among community-dwelling older women. Australian \& New Zealand Journal of Psychiatry, 38(11-12), 940-947.

Chyun, D. A., Melkus, G. D., Katten, D. M., Price, W. J., Davey, J. A., Grey, N., \& Wackers, F. J. T. (2006). The association of psychological factors, physical activity, neuropathy, and quality of life in type 2 diabetes. Biological research for Nursing, 7(4), 279-288.

Dubuc, M. M., Aubertin-Leheudre, M., \& Karelis, A. D. (2020). Lifestyle habits predict academic performance in high school students: The adolescent student academic performance longitudinal study (ASAP). International journal of environmental research and public health, $17(1), 243$.

Erickson, K. I., Hillman, C., Stillman, C. M., Ballard, R. M., Bloodgood, B., Conroy, D. E., \& Powell, K. E. (2019). Physical activity, cognition, and brain outcomes: a review of the 2018 physical activity guidelines. Medicine and science in sports and exercise, 51(6), 1242.

Freire, R. S., Lélis, F. L. D. O., Fonseca, J. A. D., Nepomuceno, M. O., \& Silveira, M. F. (2014). Prática regular de atividade física: estudo de base populacional no Norte de Minas Gerais, Brasil. Revista Brasileira de Medicina do Esporte, 20, 345-349.

Gomez-Pinilla, F., \& Hillman, C. (2013). The influence of exercise on cognitive abilities. Comprehensive Physiology, 3(1), 403.

Hall, G., Laddu, D. R., Phillips, S. A., Lavie, C. J., \& Arena, R. (2021). A tale of two pandemics: How will COVID-19 and global trends in physical inactivity and sedentary behavior affect one another? Progress in cardiovascular diseases, 64, 108.

Hallal, P. C., Matsudo, S. M., Matsudo, V. K., Araújo, T. L., Andrade, D. R., \& Bertoldi, A. D. (2005). Physical activity in adults from two Brazilian areas: similarities and differences. Cadernos de saude publica, 21, 573-580.

Li, J. W., O'Connor, H., O'Dwyer, N., \& Orr, R. (2017). The effect of acute and chronic exercise on cognitive function and academic performance in adolescents: A systematic review. Journal of science and medicine in sport, 20(9), 841-848.

Malta, D. C., Szwarcwald, C. L., Barros, M. B. D. A., Gomes, C. S., Machado, Í. E., Souza, P. R. B. D., \& Gracie, R. (2020). A pandemia da COVID-19 e as mudanças no estilo de vida dos brasileiros adultos: um estudo transversal, 2020. Epidemiologia e Serviços de Saúde, 29.

Matsudo, S., Araújo, T., Matsudo, V., Andrade, D., Andrade, E., \& Oliveira, L. C. Questionário Internacional de Atividade Fisica (IPAQ): Estudo de validade e reprodutibilidade no Brasil. Atividade Física e Saúde 2001; 6 (2): 5-18.

Mummery, K., Schofield, G., \& Caperchione, C. (2004). Physical activity: Physical activity dose-response effects on mental health status in older adults. Australian and New Zealand journal of public health, 28(2), 188-192.

Olson, S. H., Iyer, S., Scott, J., Erez, O., Samuel, S., Markovits, T., \& Kurtz, R. C. (2005). Cancer history and other personal factors affect quality of life in patients with hepatitis C. Health and quality of life outcomes, 3(1), 1-7. 
Research, Society and Development, v. 10, n. 17, e184101724766, 2021 (CC BY 4.0) | ISSN 2525-3409 | DOI: http://dx.doi.org/10.33448/rsd-v10i17.24766

Pucci, G. C. M. F., Rech, C. R., Fermino, R. C., \& Reis, R. S. (2012). Associação entre atividade física e qualidade de vida em adultos. Revista de Saúde Pública, 46, 166-179.

Sibley, B. A., \& Etnier, J. L. (2003). The relationship between physical activity and cognition in children: a meta-analysis. Pediatric exercise science, 15(3), $243-256$.

Tortella, G. (2008). Teste de Atenção por cancelamento: avaliação da atenção em estudantes do ensino fundamental. Avaliaçao Psicologica: Interamerican Journal of Psychological Assessment, 7(2), 265-267.

Watson, A., Timperio, A., Brown, H., Best, K., \& Hesketh, K. D. (2017). Effect of classroom-based physical activity interventions on academic and physical activity outcomes: a systematic review and meta-analysis. International Journal of Behavioral Nutrition and Physical Activity, 14(1), 1-24

Yan, H., Zhang, R., Oniffrey, T. M., Chen, G., Wang, Y., Wu, Y., \& Moore, J. B. (2017). Associations among screen time and unhealthy behaviors, academic performance, and well-being in Chinese adolescents. International journal of environmental research and public health, 14(6), 596. 